\title{
Towards New Chiroptical Transitions Based on Thought Experiments and Hypothesis
}

\author{
Takashiro Akitsu *, Sanyobi Kim and Daisuke Nakane
}

check for updates

Citation: Akitsu, T.; Kim, S.; Nakane,

D. Towards New Chiroptical

Transitions Based on Thought Experiments and Hypothesis.

Symmetry 2021, 13, 1103.

https://doi.org/10.3390/

sym 13061103

Academic Editor: György Keglevich

Received: 20 May 2021

Accepted: 10 June 2021

Published: 21 June 2021

Publisher's Note: MDPI stays neutral with regard to jurisdictional claims in published maps and institutional affiliations.

Copyright: (c) 2021 by the authors. Licensee MDPI, Basel, Switzerland. This article is an open access article distributed under the terms and conditions of the Creative Commons Attribution (CC BY) license (https:/ / creativecommons.org/licenses/by/ $4.0 /)$.
Department of Chemistry, Faculty of Science, Tokyo University of Science, 1-3 Kagurazaka, Shinjuku-ku, Tokyo 162-8601, Japan; 1320543@ed.tus.ac.jp (S.K.); a29776@rs.tus.ac.jp (D.N.)

* Correspondence: akitsu2@rs.tus.ac.jp; Tel.: +81-3-5228-8271

\begin{abstract}
We studied supramolecular chirality induced by circularly polarized light. Photoresponsive azopolymers form a helical intermolecular network. Furthermore, studies on photochemical materials using optical vortex light will also attract attention in the future. In contrast to circularly polarized light carrying spin angular momentum, an optical vortex with a spiral wave front and carrying orbital angular momentum may impart torque upon irradiated materials. In this review, we summarize a few examples, and then theoretically and computationally deduce the differences in spin angular momentum and orbital angular momentum depending on molecular orientation not on, but in, polymer films. UV-vis absorption and circular dichroism (CD) spectra are consequences of electric dipole transition and magnetic dipole transition, respectively. However, the basic effect of vortex light is postulated to originate from quadrupole transition. Therefore, we explored the simulated CD spectra of azo dyes with the aid of conventional density functional theory (DFT) calculations and preliminary theoretical discussions of the transition of CD. Either linearly or circularly polarized UV light causes the trans-cis photoisomerization of azo dyes, leading to anisotropic and/or helically organized methyl orange, respectively, which may be detectable by CD spectroscopy after some technical treatments. Our preliminary theoretical results may be useful for future experiments on the irradiation of UV light under vortex.
\end{abstract}

Keywords: supramolecular chirality; DFT; circular dichroism; transition; angular momentum

\section{Introduction}

Photo-induced molecular orientation due to the trans-cis isomerization of an azobenzene derivative moiety, namely, linearly or circularly polarized light-induced optical anisotropy or helical alignment, respectively, attributed to molecular reorientation caused by the Weigert effect, has been extensively studied [1,2] (Figure 1). In the field of polymer chemistry in particular, studies on supramolecular chirality focus on the origin of chirality, and many papers have been reported to date $[3,4]$.

Besides pure azo-polymers [5], our research group and others have explored metal complexes aiming to develop new functional materials based on aligned isolated metal complexes co-existing [6-8] or azobenzene derivatives [9-11], in a matrix or on the surfaces of various materials. Metal complexes undertake electric dipole transition when excited by light absorption [12], and azobenzene derivatives absorb the specific orientation of the light irradiated against molecular axes to carry out trans-cis photoisomerization [13].

Therefore, spectroscopic measurements and computational chemistry techniques have been developed for the photo-orientation of azo metal complexes. Schiff base metal complexes, as well as azobenzene moieties dispersed in a polymer matrix, increase optical anisotropy after the irradiation of linearly or circularly polarized ultraviolet (UV) light, a process that can be observed using various spectroscopic measurements. Polarized UV-vis (visible) [14,15], IR [16,17], or circular dichroism (CD) [18,19] spectra can now be simulated via the energy of the electronic transitions using conventional computational calculations $[20,21]$. 


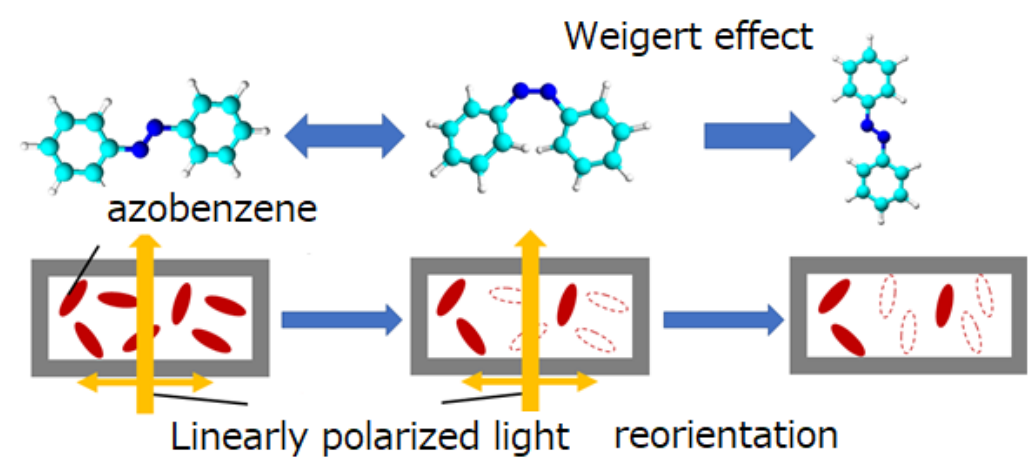

Figure 1. Weigert effect of azobenzene showing cis-trans photoisomerization in a polymer matrix (gray squares) resulting from linearly polarized UV light (large yellow arrow and small yellow arrow are the electric vector of polarized light and the molecular orientation, respectively).

In recent years, studies on optical vortex light have attracted much attention as a new kind of chiral light [22-24]. In contrast to circularly polarized light carrying spin angular momentum, a light vortex exhibiting a spiral wave-front and carrying orbital angular momentum may mechanically impart torque when irradiated onto an object (Figure 2) [25-27]. Indeed, some examples of spiral damage on the "surface" of azobenzenecontaining polymers have been reported [28,29]. In this context, we should consider excitation via light absorption or electronic transitions in a molecule, which cannot be observed in the case of ordinary or conventional lights [30,31]. However, new theoretical approaches to interpreting light absorption should be developed for such new types of light, except for the spin of magnets, for which techniques have already been developed [32,33].
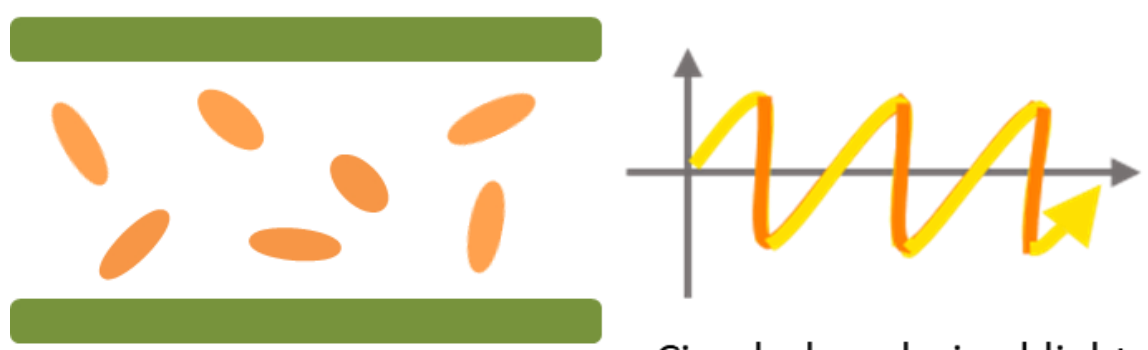

Circularly polarized light

(a)
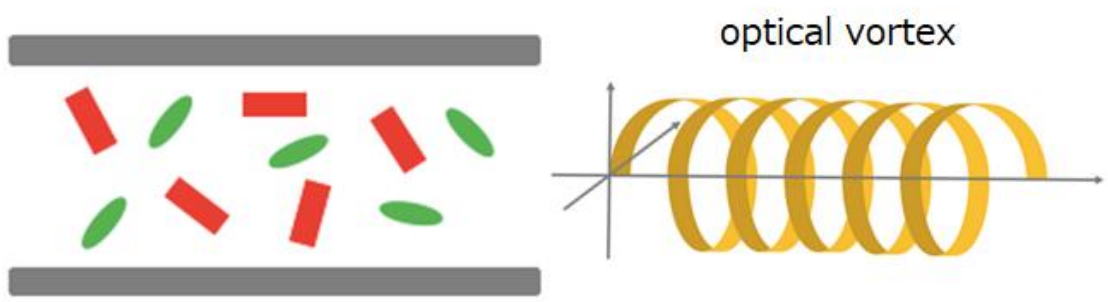

(b)

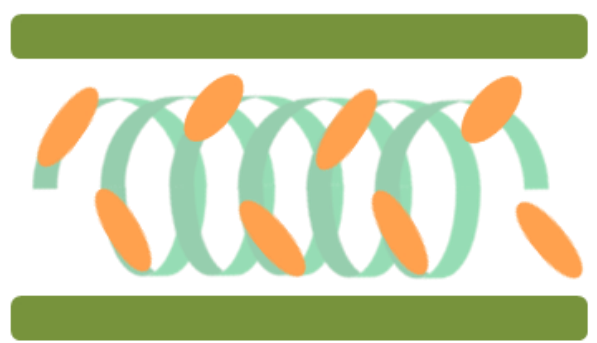

\section{,}


in the case of optical vortex light.) and hypothesis frameworks that are analogous with conventionally established treatments [34,35]. We are seeking to determine what will happen to the metal complexes and azo-groups in the polymer medium [36-38] when the light vortex is irradiated. All actual experiments on conventional light were carried out in the referenced papers using cast films less than $0.05 \mathrm{~mm}$ thick at room temperature.

\section{Thought Experiments of Optical Vortex Applied for Some Cases of Known Systems}

\subsection{Separated Azobenzene and Schiff Base Metal Complexes}

First, it is easy to imagine facing technical problems when using $C D$ to determine the orientation of molecules $[39,40]$ that are not related to the optical vortex, which may induce chiral supramolecular orientation [41,42]. Even if using circularly polarized light appropriately generated by a conventional UV light source with optical devices (Figure 3), supramolecular helical chirality was inferred from so-called artifact CD peaks due to the $\mathrm{LD}$ and $\mathrm{CD}$ terms in the oriented samples [43]. Although several experimental methods for overcoming or avoiding this issue have been proposed [44,45], the rotation and averaging of the CD spectra of oriented samples may be one of the easiest solutions (Figure 3).

\section{Irradiation of elliptically polarized light to film}
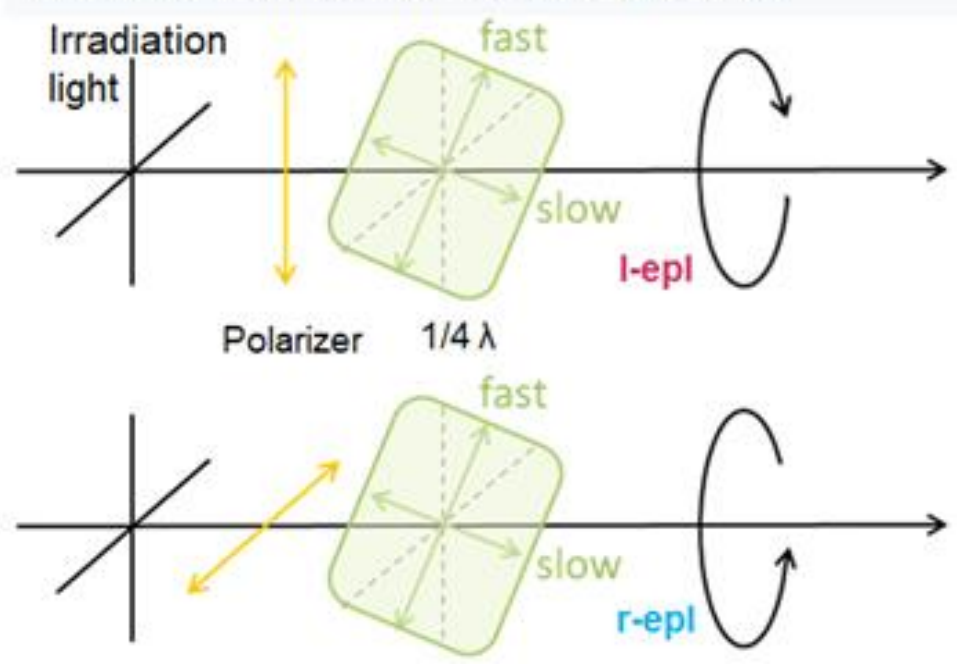

(a)

\section{Measurement of $C D$ spectra}

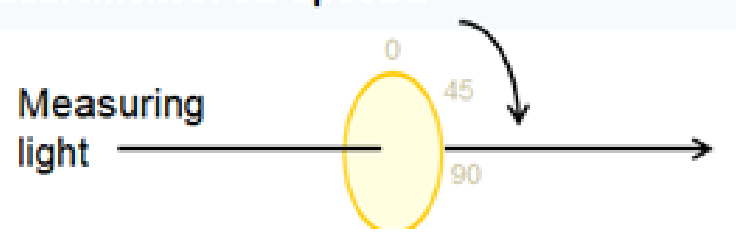

(b)

Figure 3. (a) Generation of left-(l-) and right-(r-) handed elliptically polarized light (epl) using a conventional elongated circularly polarized light source to irradiate samples. (b) Rotating measurement of CD spectra to overcome the artifact peak problem.

Diastereomers of (achiral or chiral) Schiff base $\mathrm{Ni}(\mathrm{II}), \mathrm{Cu}(\mathrm{II})$, and $\mathrm{Zn}$ (II) complexes with or without azobenzene moiety in their ligands were prepared as PMMA cast films (namely organic/inorganic photofunctional hybrid materials) (Figure 4) [46]. These samples were irradiated with linearly or circularly polarized UV light; we then compared their CD spectra before and after irradiation to confirm the molecular orientation inside the hybrid materials. Utilizing symmetry, the optically active complex is strengthened by the molecular CD and tends to form a helical pattern. Achiral complexes, on the other hand, are suitable for comparing spiral-only CD spectra. 
<smiles>OCC(c1ccccc1)N1C=Cc2cc(Cl)cc(Cl)c2OC12N=Cc1cc(Cl)cc(Cl)c1O2</smiles>

(a)

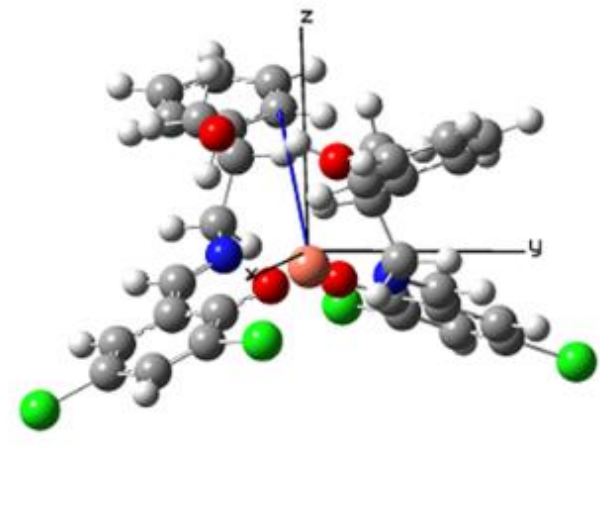

(b)

Figure 4. Molecular (a) and DFT-optimized (b) structures of the Schiff base Ni(II), Cu(II), and Zn(II) complexes without azobenzene moieties. These complex and azobenzene molecules were dispersed in a PMMA film.

Using wavelength selective UV light may also help to overcome this issue [47]. In the first step, we saw that circularly polarized UV light induced different chiral supramolecular orientations, as assessed by CD spectra, before and after UV irradiation. In the second step, we confirmed that the linearly polarized UV light induced little changes in CD spectra via control experiments. Circularly polarized UV light at 260,318 , and $380 \mathrm{~nm}$ was irradiated for $0,3,5$, and $8 \mathrm{~min}$. The differences in the CD spectra (considered blank data to remove the effect artifact peaks) indicated increasing CD peaks, suggesting that chiral supramolecular orientation was induced. Light at $260 \mathrm{~nm}$ was effective for inducing bands at about $300 \mathrm{~nm}$ in all complexes (in addition, the band at $400 \mathrm{~nm}$ was induced only for the $\mathrm{Zn}$ (II) complex). Light at 318 and $380 \mathrm{~nm}$ was effective at inducing bands at about 300 and $500 \mathrm{~nm}$ for all complexes (in addition, the band at about $500 \mathrm{~nm}$ was induced for the $\mathrm{Ni}$ (II) and $\mathrm{Zn}$ (II) complexes, and was induced at about $700 \mathrm{~nm}$ for the $\mathrm{Cu}$ (II) complex).

On the other hand, similar differences in the CD spectra before and after linearly polarized UV light irradiation at selective wavelengths were observed; that is, no CD peaks could be observed in the long-wavelength region without noise peaks. Generally, in solutions with isotropic environments, proper CD spectra can be obtained for chiral metal complexes, which has been ascribed to molecular chirality. That said no CD spectra could be observed for achiral metal complexes. On the other hand, it is well known that samples manifesting an anisotropic environment (for example, solid state or well-oriented films) show not only proper $C D$ peaks but also artifact $C D$ peaks. In the different $C D$ spectra, the artifact peaks were cancelled out when no changes related to chirality occurred, even if molecular or supramolecular chirality was present. In other words, although linearly polarized UV light induced optical anisotropy in metal complexes in hybrid materials, the supramolecular chiral order was not affected, even in chiral metal complexes.

In conclusion, the presence of $C D$ peaks in different $C D$ spectra is attributed to the supramolecular helical orientation induced by circularly polarized UV light irradiation, for achiral as well as chiral metal complexes. Additionally, UV light of specific wavelengths can effectively induce molecular orientation.

\subsection{Azobenzene Containing Schiff Base Metal Complexes}

Photofunctional materials sensitive to optical vortex UV light will also attract attention in the near future. Until then, both experimental results and computational treatments carried out with conventional polarized light and azobenzene-related compounds should 
be thoroughly examined, and their difference from the optical vortex should be considered [48,49]. Clear circularly polarized light experimental data (identical to conventional circularly polarized light irradiation) are useful for light absorption treatments (unknown for optical vortex), and affect the CD spectra.

We also employed azo-containing mononuclear Schiff base metal complexes in PMMA films for assessing the molecular orientation effect induced by several types of UV light irradiation (Figure 5) [50,51]. Among such complexes, we selected a complex with $\mathrm{M}=\mathrm{Zn}$ (II) and $\mathrm{R}=\mathrm{H}$, which manifested an intense peak at $380 \mathrm{~nm}$, for the photoirradiation experiments. We also confirmed the cis-trans photoisomerization of the azo-moiety of the ligand with wavelength-selective UV light irradiation, the Weigert effect, which is the linear polarization of anisotropic molecular orientation induced by light, and supramolecular chiral arrangement induced by circularly polarized light irradiation. Employing a conventional laboratory light source, linearly polarized and circularly polarized UV light was used, and the rotating CD spectra of the complexes were measured (Figure 5). The peaks may be attributed to supramolecular chiral arrangement induced by circularly polarized light irradiation.

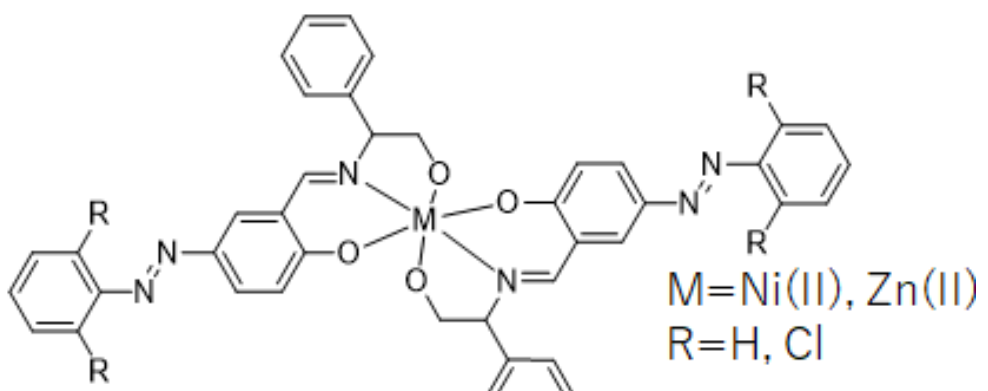

(a)

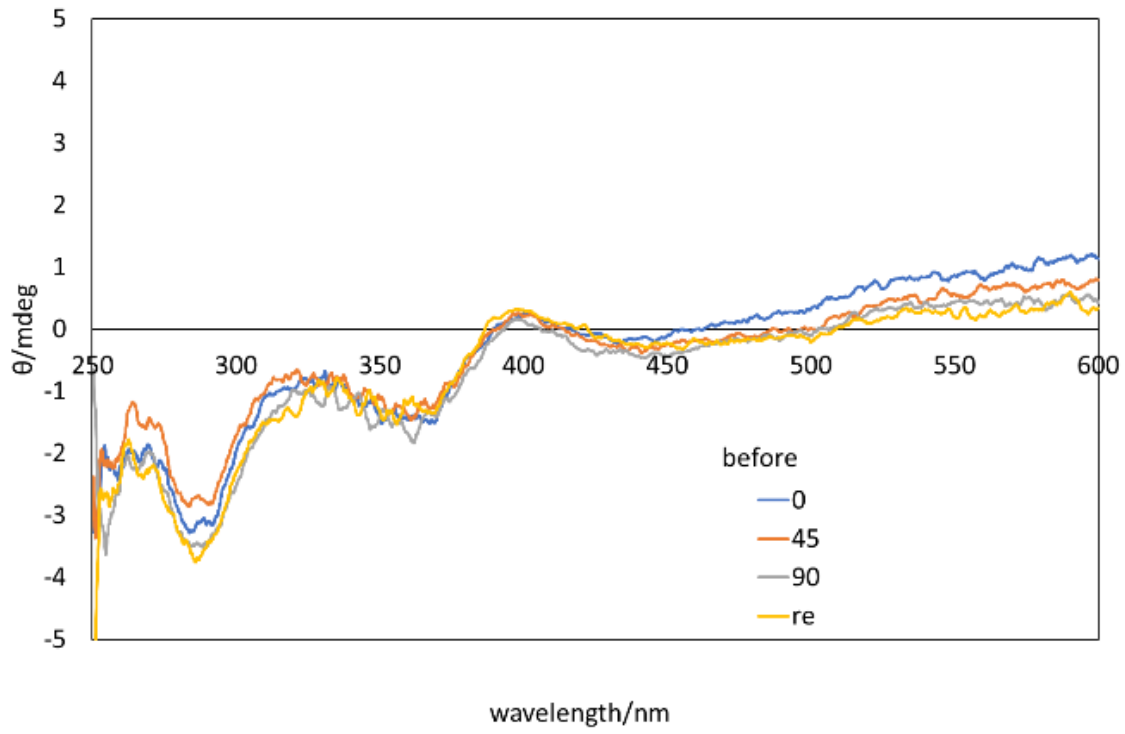

(b)

Figure 5. Molecular structures of the chiral Schiff base Ni(II) and Zn(II) complexes with azobenzene moiety (a) and rotating CD spectra measured at four points in the PMMA films after circularly polarized UV light irradiation (b). Experiments using conventionally polarized UV light have been undertaken in the corresponding literature, and the more rigid $\mathrm{M}=\mathrm{Zn}$ (II) complex $\mathrm{R}=\mathrm{H}$ will be used for the thought experiment. 


\subsection{Mechanical Effect of the Light for Complexes without Azobenzene}

Although it was not clear in our experiments, circularly polarized light is expected to have a physical and mechanical effect on the distribution of singular points and peripheral parts of the optical vortex $[52,53]$. Azobenzene polymers have several different mechanical properties $[54,55]$. Without azo-groups, the typical Weigert effect does not occur. However, it may be a useful tool for discussing the direct mechanical effects (twisting) of light [56,57].

We prepared organic/inorganic hybrid materials composed of water-soluble binuclear Zn(II) Schiff base complexes without azo-compounds in a PVA film to investigate changes to molecular orientation induced by linearly and circularly polarized UV light (Figure 6) $[58,59]$. The data showing the variation in Figure 6 reflect the reality better than other low-quality images. This is because a slight orientation change induced by polarized UV light was measured for a sample containing no azobenzene moiety, indicating a direct photoisomerization response. We have synthesized and characterized a new Schiff base dinuclear $\mathrm{Zn}$ (II) complex including chloride anions as axial ligands, and prepared PVA polymer cast films. Without photochromic additives, optical dichroism at charge transfer peaks could be observed after linearly polarized UV light irradiation with electric field vectors, illustrated by the increasing anisotropy of the molecular orientation of the complexes. For example, the related $\mathrm{Ni}$ (II) complex showed the most obvious anisotropy after linearly polarized UV light irradiation for $10 \mathrm{~min}$. Figure 6 also shows the angular dependence of the polarized UV-vis spectra (absorbance at $367 \mathrm{~nm}$ ) of the $\mathrm{Ni}(\mathrm{II})$ complex. The Zn(II) complex also manifested similar behavior to the $\mathrm{Ni}$ (II) complex. Compared with other CD data from control experiments (only PVA film or hybrid materials, solutions or film, wavelengths or the orbital angular moment dependence of UV light), we derived preliminary evidence that circularly polarized UV light induced the molecular orientation of the $\mathrm{Zn}(\mathrm{II})$ complex in hybrid materials.

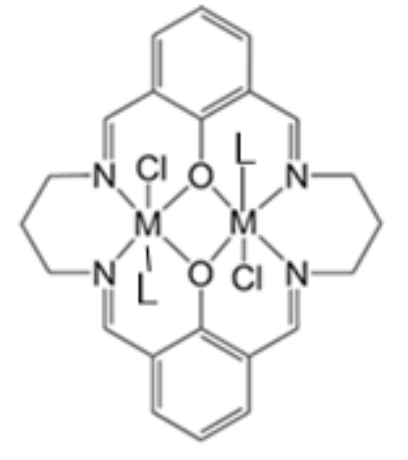

(a)

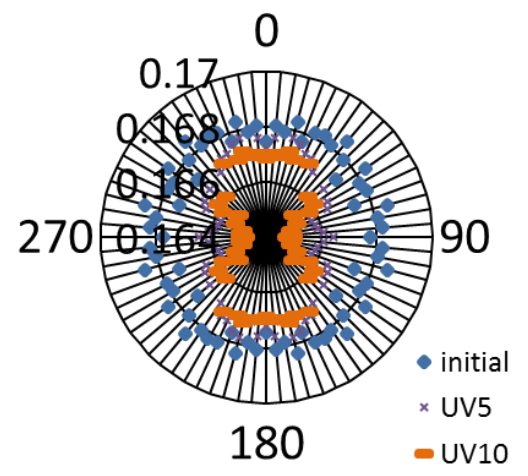

(b)

Figure 6. (a) Molecular structures of the Schiff base $\mathrm{Zn}$ (II) complexes without azobenzene $(\mathrm{M}=\mathrm{Ni}$, $\mathrm{Zn} ; \mathrm{L}=\mathrm{H}_{2} \mathrm{O}$ or $\mathrm{OH}$ of PVA (expected axial ligands)). (b) Angular dependence of absorbance (namely, $0.17,0.168,0.166$, and 0.164 of the radial circles) of polarized UV-vis spectra ( $367 \mathrm{~nm}$ ) of Ni(II) complex in PVA film after linearly polarized UV light irradiation (<350 nm) for 0 min (initial), 5 min (UV 5) and $10 \mathrm{~min}$ (UV 10). PVA films composed of only metal complexes or metal complexes and azo-dyes were used for comparison.

For considering the mechanical effects of optical vortices by comparison with spin and orbital angular momentum [60], the following data handling methods can be used (this is a thought experiment). Consider the following situation: a PMMA film containing a complex with an extended planar ligand and having azobenzene moiety [61] is irradiated with a linearly polarized optical vortex with an orbital angular momentum $1=-1$, 1 near the absorption wavelength. The measurement points for each wavelength of the $\mathrm{CD}$ spectrum, measured by changing the angle, are fitted with the sine curve equation $y=A \sin \{(x B) \cdot \pi / 90\}+C$, and the graph of the A term is constructed by the linearly polarized light component of the optical vortex. The graph of the $C$ term can thus be analyzed 
by the orbital angle momentum. The graph of the linearly polarized light component A shows a periodic change, suggesting the formation of chiral orientation. Further, in the graph of the $C$ term depicting the orbital angular momentum, the influence of the angle-independent component is small, and a half-wavelength deviation in the orbital angular momentum is expected, with different signs in specific wavelength regions. The appearance is different depending on the surface [62], and in the molecule inside the material (where electronic states are important [63]), the orbital angular momentum of the optical vortex may have a physical and mechanical influence on the distribution of the singular point and the peripheral part of the optical vortex, or the polarization of light [64].

\section{Theoretical Consideration of Topological Factors for CD}

The theory of CD spectroscopy (rotation of polarized light by chiral materials) is well-established $[65,66]$. The spatial factors of both chiral materials and electromagnetic waves $[67,68]$ and the resulting molecular orientation [69] should be considered, except for electron transitions, during excitation [70]. Polarized light is light in which the vibration is fixed in a specific direction. Let us set the coordinates of $\mathrm{x}$ and $\mathrm{y}$ on the vibrating surface and try to decompose the components. When the two are in the same phase, the light will be linearly polarized. As the phase shifts, the light becomes circularly polarized or elliptically polarized. The equation for the vibration of linearly polarized light in the $\mathrm{x}$ and $\mathrm{y}$ directions is $\cos \omega \mathrm{t}$. Left-handed polarized light is achieved by shifting the phase by $\pi / 2$ in only one direction, and right-handed polarized light involves shifting by $\pi$.

$$
\begin{aligned}
& \text { Linearly polarization light } x=\cos \omega t y=\cos \omega t \\
& \text { Left polarization light } x=\cos \omega t y=\cos (\omega t+\pi / 2) \\
& \text { Right polarization light } x=\cos \omega t y=\cos (\omega t+\pi)
\end{aligned}
$$

where $\omega$ and $t$ denote angular frequency and time, respectively. This is a commonly used treatment in which circularly polarized light (elliptical polarized light) is derived from the phase difference.

The vibration equations are also shown above, where $\omega$ represents the frequency.

Since the synthetic electric field of left and right circularly polarized light is a composite of sine and cosine, it is convenient to mathematically display it as a complex number.

The time-varying part of the Hamiltonian of electric charge in the electromagnetic field is taken out and used as the perturbation term. It is shown in Equation (4).

$$
H^{\prime}=-\sum_{j} \frac{e}{m_{j} c} A^{j} \cdot P^{j}
$$

where $j$ : respective charge in molecule; m: mass; $c$ : light velocity; $e$ : elementary charge; $A$ : vector potential; $P$ : momentum.

Since the conventional method of confirming that the vector potential is uniform on the molecule is coarse, the value of the potential changes according to the position on the molecule. Furthermore, when the matrix element is calculated by substituting the Taylor expansion of the vector potential into Equation (4), the electric and magnetic dipole moments appear, as shown in Equation (5):

$$
\left\langle\varphi^{\prime}\left|H^{\prime}\right| \varphi\right\rangle=-\left\{\frac{i \Delta E}{c h}\left\langle\varphi^{\prime}\left|\mu_{e}\right| \varphi\right\rangle \cdot A^{0}+\left\langle\varphi^{\prime}\left|\mu_{m}\right| \varphi\right\rangle \cdot(\nabla \times A)\right\} \exp \left(i \frac{2 \pi \Delta E}{h} t\right)
$$

where $\varphi^{\prime}, \varphi$ : different kind of electronic state; $\Delta E$ : energy gap between two states; $h$ : Planck's constant; $\mu_{e}$ : operator of electronic dipole moment; $\mu_{m}$ : operator of magnetic dipole moment.

The wave function in the perturbed state is expressed as a linear combination of eigenfunctions that are strictly solved, and Equation (5) is used to obtain the coefficient.

Finally, the induced electric and magnetic dipole moment can be derived. 
The details are omitted, but the results are shown below.

$$
\begin{aligned}
& \mu_{e}^{\prime}=\alpha E^{\prime}+\gamma H^{\prime}-\frac{\beta}{c} \frac{d H^{\prime}}{d t} \\
& \mu_{m}^{\prime}=k H^{\prime}+\gamma E^{\prime}-\frac{\beta}{c} \frac{d E^{\prime}}{d t}
\end{aligned}
$$

where $\mu_{e}^{\prime}$ : induced electric dipole moment; $\mu_{m}^{\prime}$ : induced magnetic moment; $E^{\prime}$ and $H^{\prime}$ : electric and magnetic fields that actually act on the molecule, not the external field.

The coefficient of the differential term in Equations (6) and (7) is important in the discussion of optical rotation.

$$
\begin{gathered}
\beta=\frac{c}{3 \pi h} \sum_{n} \frac{\operatorname{Im}\left\{\left\langle 0\left|\mu_{e}\right| n\right\rangle \cdot\left\langle n\left|\mu_{m}\right| 0\right\rangle\right\}}{v_{n 0}-v^{2}} \\
R=\operatorname{Im}\left\{\left\langle 0\left|\mu_{e}\right| n\right\rangle \cdot\left\langle n\left|\mu_{m}\right| 0\right\rangle\right\}
\end{gathered}
$$

where 0 : background state; $v_{n 0}$ : frequency between $n$ and $0 ; v$ : frequency of external light.

Equation (9) indicates rotational strength, and it is part of Equation (8).

The vector potential of the magnetic field created by a small loop current is proportional to the product of the current and the area of the loop, which is referred to as the magnetic dipole moment. The orientation of the vector is perpendicular to the loop plane. As shown in Equation (9), the rotational intensity is the inner product of the electric dipole moment and the magnetic dipole moment. Assuming that the angle formed by these is $0^{\circ}$, when the linear motion of the electron and the loop rotation are added, a spiral trajectory is drawn. This is why CD measurements are used to confirm the spiral orientation formed by circularly polarized light. On the other hand, the light vortex exhibits a spiral wavefront and carries orbital angular momentum, in which the electric vector is distributed in complex directions [71].

\section{Computational Simulation}

Our understanding of the chirality in the interaction between matter and light has deepened in recent years. Moreover, the UV-vis and CD spectra are observed as consequences of electric dipole transition and magnetic dipole transition, respectively. However, the inherent effect of vortex light is postulated to originate from quadrupole transition. Therefore, the simulated CD spectra of both isomers of methyl orange ( $\mathrm{MO}, p$ $\left(\mathrm{CH}_{3}\right)_{2} \mathrm{NC}_{6} \mathrm{H}_{5}-\mathrm{N}=\mathrm{N}-\mathrm{C}_{6} \mathrm{H}_{5}-\mathrm{SO}_{3}{ }^{-}$) [61], which is a typical azo-dye, can be extracted easily by DFT calculation, namely quadrupole transitions (Figure 7). When irradiating with an optical vortex of $280 \mathrm{~nm}$, which is very weak, no chiral order is formed in the trans form of MO. However, in the case of the cis form of MO, a chiral molecular orientation was formed during the winding after optical vortex irradiation by comparison with $\mathrm{MO}$ using the TD-DFT calculation. These results are similar to the corresponding experimental results derived using circularly polarized UV light.

Furthermore, a new CD peak appears when a PVA composite film containing MO and an achiral dinuclear Zn(II) complex (Figure 8) [58,59] is irradiated with an optical vortex. Here, we employed water-soluble polymer PVA and metal complexes in our conventional polarized light experiments to expand the protein matrix. Peaks with the same sign appeared on the front and back of the film, and the peaks become symmetrical (viewed from both sides) $[60,61]$ when the orbital angular momentum of the optical vortex became $1=-1,1$ (Figure 8 ). The formation of the chiral molecular orientation order of the complex can be simulated using DFT calculations. 


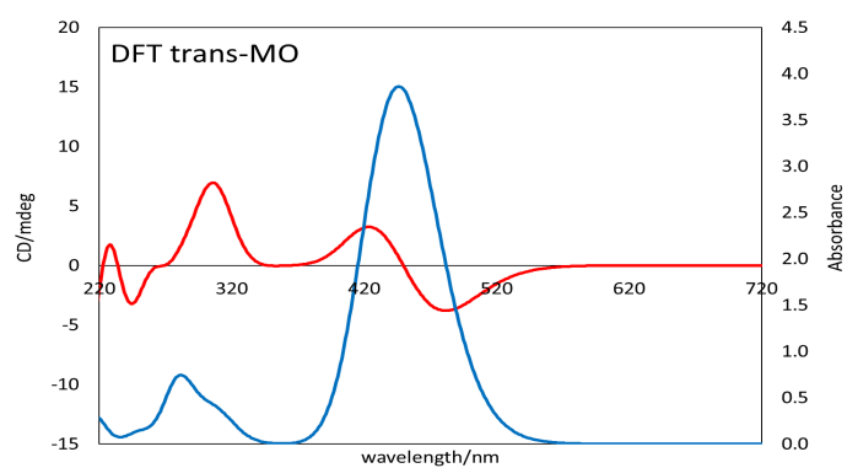

(a)

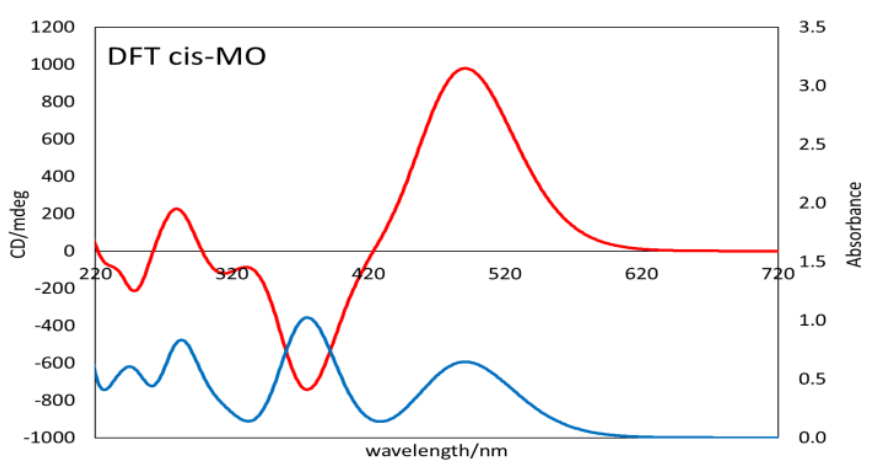

(b)

Figure 7. Simulated UV-vis (blue) and CD (red) spectra of MO for the trans form (a) and the cis form (b) using the TD-DFT calculation via Gaussian09. As for the azo-group $(-\mathrm{N}=\mathrm{N}-)$, the cis and trans isomers can be defined for MO, showing a short wavelength shift of the strongest peak of UV-vis spectrum. The CD spectrum of "achiral" MO isomers can be simulated using TD-DFT to distinguish supramolecular chirality due to the helical arrangement of MO molecules.

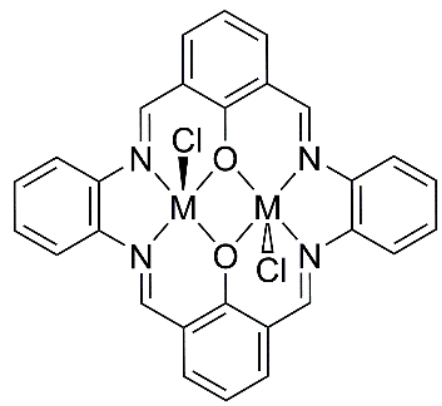

(a)

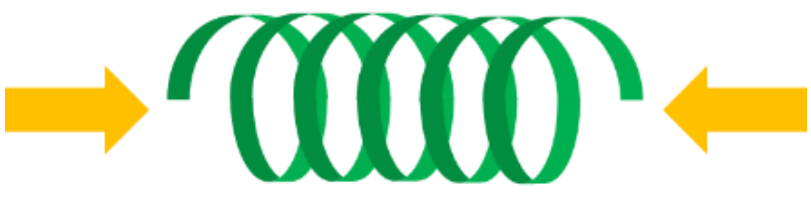

(b)

Figure 8. (a) Molecular structures of the dinuclear Schiff base $\mathrm{Zn}(\mathrm{II})$ complex $\left(\mathrm{ZnL}_{2}\right)$ dispersed with MO. (b) If a positive peak can be obtained regardless of whether the CD peak is measured from the front or back side, this is because the structure is the same from both perspectives. For example, if a spiral structure was formed, it would be "left-handed" when viewed from either side. The Green helical shape denotes molecular arrangement induced by light. Yellow arrows depict viewpoints from both sides.

In the case of Figure 8, the MO directly underwent light-induced changes in its molecular orientation, giving rise to the molecular orientation of a metal complex. The effect of direct light-induced molecular orientation change in a metal complex may be weaker than the effect in an MO. In both situations, helically aligned molecules dispersed in a PVA film can exhibit CD signals due to the supramolecular helical chirality of chiral or achiral molecules.

Various changes after optical vortex UV irradiation were assessed. The sample for this simulation was irradiated with two types of optical vortices; the maximum absorption wavelength of the complex was $253 \mathrm{~nm}$ and $360 \mathrm{~nm}$ and the orbital angular momentum $(1=-1,1)$ of the wavelength not absorbed was $280 \mathrm{~nm}$. The change in the CD spectrum before and after the irradiation was estimated. It increased to around $280 \mathrm{~nm}$, which was the same as the wavelength $(280 \mathrm{~nm})$, but this was not because the MO in the PVA film transitioned from the trans form to the cis form. The torque of the optical vortex causes the $\mathrm{Zn}$ (II) complex to form a spirally twisted chiral order. In addition, irradiation with an optical vortex $(l=1)$ under the same conditions increased the CD spectrum, as in the case of $(1=-1)$, and its peak dipped downwards. This suggests that the angular momentum of the optical vortex has a physical effect on the molecules in the polymer films. 


\section{Conclusions}

We have summarized the available information on, and discussed the photo-induced orientation of, azo-dyes and metal complexes in polymer films. The future prospects of such studies are as follows. Firstly, they have potential applicability in materials whose anisotropically or helically aligned physical properties can be controlled by light, that is, in optically switchable materials. Nowadays, studies on smart materials using polymers are attracting attention, and highly efficient and high-performance devices are required. On the other hand, metal complexes have various physical properties, such as magnetism, optical properties, electron transfer, etc., which depend on the metal ions' and ligands' properties. Our study may be useful for determining the functions of metal complexes as induced by various types of light. The second aspect is the manipulation of chiral orientation achieved by left and right circularly polarized light or optical vortex, if possible. The chiral orientation of solid-state soft material polymers with an azobenzene moiety on the side chain has been explored. However, there are few studies on the chiral orientation of metal complexes present in (but not on) a polymer matrix. At present, we still have some problems about the interaction between optical vortex and substances. The improvement of measurement methods and actual experiments is necessary for this theoretical study. As an example, local CD observation using a condensing system in a CD spectrometer can be considered. The optical vortex is a so-called "donut-shaped" spiral light, and this may also be beneficial to spatial resolution, along with depth direction and beam size. In composite systems such as the ones mentioned, the difference in the effect on the substance at the central singularity point and in other parts has not been clarified. Analysis in the nano-scale range (volume) will be important for investigating the effects of an optical vortex on a substance, other than the electronic states in a molecule.

Author Contributions: Writing paper, T.A. and S.K. Checking manuscript, D.N. All authors have read and agreed to the published version of the manuscript.

Funding: This research received no external funding.

Institutional Review Board Statement: Not applicable.

Informed Consent Statement: Not applicable.

Data Availability Statement: The data presented in this study are openly available in the article.

Acknowledgments: The author would like to express sincere thanks to Michiya Fujiki (NAIST) and Masahiro Katoh and Masaki Fujimoto (UVSOR, Institute for Molecular Science).

Conflicts of Interest: The authors declare no conflict of interest.

\section{References}

1. Natansohn, A.; Rochon, P. Photoinduced Motions in Azo-Containing Polymers. Chem. Rev. 2002, 102, 4139-4175. [CrossRef]

2. Ichimura, K. Photoalignment of Liquid-Crystal Systems. Chem. Rev. 2000, 100, 1847-1874. [CrossRef] [PubMed]

3. Fujiki, M. Supramolecular Chirality: Solvent Chirality Transfer in Molecular Chemistry and Polymer Chemistry. Symmetry 2014, 6, 677-703. [CrossRef]

4. Fujiki, M. Mirror Symmetry Breaking in Helical Polysilanes: Preference between Left and Right of Chemical and Physical Origin. Symmetry 2010, 2, 1625-1652. [CrossRef]

5. Li, S.; Feng, Y.; Long, P.; Qin, C.; Feng, W. The light-switching conductance of an anisotropic azobenzene-based polymer close-packed on horizontally aligned carbon nanotubes. J Mater. Chem. C 2017, 5, 5068-5075. [CrossRef]

6. Akitsu, T.; Itoh, T. Polarized spectroscopy of hybrid materials of chiral Schiff base cobalt(II), nickel(II), copper(II), and zinc(II) complexes and photochromic azobenzenes in PMMA films. Polyhedron 2010, 29, 477-487. [CrossRef]

7. Aritake, Y.; Akitsu, T. The role of chiral dopants in organic/inorganic hybrid materials containing chiral Schiff base Ni(II), $\mathrm{Cu}(\mathrm{II})$, and $\mathrm{Zn}$ (II) complexes. Polyhedron 2012, 31, 278-284. [CrossRef]

8. Yamazaki, A.; Akitsu, T. Polarized spectroscopy and polarized UV light-induced molecular orientation of chiral diphenyl Schiff base $\mathrm{Ni}(\mathrm{II})$ and $\mathrm{Cu}(\mathrm{II})$ complexes and azobenzene in a PMMA film. RSC Adv. 2012, 2, 2975-2980. [CrossRef]

9. Cantatore, T.; Granucci, G.; Persico, M. The Photo-orientation of azobenzene in viscous solutions, simulated by a stochastic model. Phys. Chem. Chem. Phys. 2014, 16, 25081-25092. [CrossRef] 
10. Bogdanov, A.V.; Vorobiev, A.K. Photo-Orientation of Azobenzene-Containing Liquid-Crystalline Materials by Means of Domain Structure Rearrangement. J. Phys. Chem. B 2013, 117, 13936-13945. [CrossRef]

11. Kimizuka, N.; Kawasaki, T.; Kunitake, T. Spectral Characteristics and Molecular Orientation of Azobenzene-Containing HydrogenBond-Mediated Bilayer Membranes. Chem. Lett. 1994, 23, 1399-1402. [CrossRef]

12. Hestand, N.J.; Spano, F.C. Expanded Theory of H- and J-Molecular Aggregates: The Effects of Vibronic Coupling and Intermolecular Charge Transfer. Chem. Rev. 2018, 118, 7069-7163. [CrossRef]

13. Peakra, H.M.D.; Burdette, S.C. Photoisomerization in different classes of azobenzene. Chem. Soc. Rev. 2012, 41, 1809-1825. [CrossRef]

14. Hicks, M.R.; Kowalski, J.; Rodger, A. LD spectroscopy of natural and syntetic biomaterials. Chem. Soc. Rev. 2010, 39, 3380-3393. [CrossRef] [PubMed]

15. Jameson, D.M.; Ross, J.A. Fluorescense Polariation/Anisotropy in Diagnostics and Imaging. Chem. Rev. 2010, 110, 2685-2708. [CrossRef] [PubMed]

16. Ito, M.; Akitsu, T.; Palafox, M.A. Theoretical interpretation of polarized light-induced supramolecular orientation on the basis of normal mode analysis of azobenzene as hybrid materials in PMMA with chiral Schiff base Ni(II), Cu(II), and Zn(II) complexes. J. Appl. Solut. Chem. Model. 2016, 5, 30-47. [CrossRef]

17. Wen, Y.; Yi, W.; Meng, L.; Feng, M.; Jiang, G.; Yuan, W.; Zhang, Y.; Gao, H.; Jiang, L.; Song, Y. Photochemical-Controlled Switching Based on Azobenzene Monolayer Modified Silicon (111) Surface. J. Phys. Chem. B 2005, 109, 14465-14468. [CrossRef]

18. Miao, T.; Yin, L.; Cheng, X.; Zhao, Y.; Hou, W.; Zhang, W.; Zhu, X. Chirality Construction from Preferred $\pi-\pi$ Stacks of Achiral Azobenzene Units in Polymer: Chiral Induction, Transfer and Memory. Polymers 2018, 10, 612. [CrossRef]

19. Thomas, R.; Tamaoki, N. Chirality transfer from chiral solvents and its memory in an azobenzene derivative exhibiting photoswitchable racemization. Org. Biomol. Chem. 2011, 9, 5389-5939. [CrossRef]

20. Aritake, Y.; Takanashi, T.; Yamazaki, A.; Akitsu, T. Polarized spectroscopy and hybrid materials of chiral Schiff base Ni(II), Cu(II), $\mathrm{Zn}(\mathrm{II})$ complexes with included or separated azo-groups. Polyhedron 2011, 30, 886-894. [CrossRef]

21. Akitsu, T.; Yamazaki, A.; Kobayashi, K.; Haraguchi, T.; Endo, K. Computational treatments of hybrid dye materials of azobenzene and chiral Schiff base metal complexes. Inorganics 2018, 6, 37. [CrossRef]

22. Allen, L.; Beijersbergen, M.W.; Spreeuw, R.J.C.; Woerdman, J.P. Orbital angular momentum of light and the transformation of Laguerre-Gaussian laser modes. Phys. Rev. A 1992, 45, 8185-8189. [CrossRef] [PubMed]

23. Katoh, M.; Fujimoto, M.; Kawaguchi, H.; Tsuchiya, K.; Ohmi, K.; Kaneyasu, T.; Taira, Y.; Hosaka, M.; Mochihashi, A.; Takashima, Y. Angular Momentum of Twisted Radiation from an Electron in Spiral Motion. Phys. Rev. Lett. 2017, 118, 094801. [CrossRef] [PubMed]

24. Hikosaka, Y.; Kaneyasu, T.; Fujimoto, M.; Iwayama, H.; Katoh, M. Coherent control in the extreme ultraviolet and attosecond regime by synchrotron radiation. Nat. Commun. 2019, 10, 4988. [CrossRef] [PubMed]

25. Padgett, M.; Courtial, J.; Allen, L. Light's Orbital Angular Momentum. Phys. Today 2004, 57, 35-40. [CrossRef]

26. Cameron, R.P.; Gotte, J.B.; Barnett, S.M.; Yao, A.M. Chrality and the angular momentum of light. Phil. Trans. R. Soc. A 2017, 375, 20150433. [CrossRef] [PubMed]

27. Forbes, A. Controlling light's helicity at the source: Orbital angular momentum states from lasers. Phil. Trans. R. Soc. A 2017, 375, 20150436. [CrossRef]

28. Ambrosio, A.; Marrucci, L.; Borbone, F.; Roviello, A.; Maddalena, P. Light-induced spiral mass transport in azo-polymer films under vortex-beam illumination. Nat. Commun. 2012, 3, 989. [CrossRef]

29. Watabe, M.; Juman, G.; Miyamato, K.; Omatsu, T. Light induced conch-shaped relief in an azo-polymer film. Sci. Rep. 2014, 4, 4281. [CrossRef]

30. Andrews, D.L.; Romero, L.C.D.; Babiker, M. On optical vortex interactions with chiral matter. Opt. Commun. 2004, 237, 133-139. [CrossRef]

31. Friedrich, B.; Herschbach, D. Polarization of Molecules Induced byIntense Nonresonant Laser Fields. J. Phys. Chem. 1995, 99, 15686-15693. [CrossRef]

32. Fujita, H.; Sato, M. Ultrafast generation of skyrmionic defects with vortex beam: Printing laser profiles on magnets. Phys. Rev. B 2017, 95, 054421. [CrossRef]

33. Fujita, H.; Sato, M. Encoding orbital angular momentum of light in magnets. Phys. Rev. B 2017, 95, 060407. [CrossRef]

34. Fujiki, M.; Yoshida, K.; Suzuki, N.; Zhang, J.; Zhang, W.; Zhu, X. Mirror symmetry breaking and restoration within $\mu \mathrm{m}-\mathrm{sized}$ polymer particles in optofluidic media by pumping circularly polaised light. RSC Adv. 2013, 3, 5213-5219. [CrossRef]

35. Fujiki, M.; Donguri, Y.; Zhao, Y.; Nakao, A.; Suzuki, N.; Yoshida, K.; Zhang, W. Photon magic: Chroptical polarization, depolarization, inversion, retention and switching of non-photochromic light-emitting polymers in optofludic medium. Polym. Chem. 2015, 6, 1627-1638. [CrossRef]

36. Barboza, R.; Bortolozzo, U.; Clerc, M.G.; Residori, S.; Vidal-Henriquez, E. Optical vortex induction via light-matter interaction in liquid-crystal media. Adv. Opt. Photonics. 2015, 7, 635-683. [CrossRef]

37. Bin, J.; Oates, W.S. A Unified Material Description for Light Induced Deformation in Azobenzene Polymers. Sci. Rep. 2015, 5, 14654. [CrossRef] 
38. Rananaware, A.; Samanta, M.; Bhosale, R.S.; Kobaisi, M.; Roy, B.; Bheemireddy, V.; Bhosale, S.V.; Peakyopadhyay, S.; Bhosale, S.V. PHotomodulation of fluoride ion binding through anion-p inteactions using a photoswitchable azobenzene system. Sci. Rep. 2016, 6, 22928. [CrossRef]

39. Schellman, J.; Jensen, H.P. Optical spectroscopy of oriented molecules. Chem. Rev. 1987, 87, 1359-1399. [CrossRef]

40. Kuball, H.-G.; Karstens, T.; Schonhofer, A. Optical Activity of Oriented Molecules. Chem. Phys. 1976, 12, 1-13. [CrossRef]

41. Morrow, S.M.; Bissette, A.J.; Fletcher, S.P. Transmission of chirality through space and across length scales. Nat. Nanotechnol. 2017, 12, 410-419. [CrossRef]

42. Nuckolls, C.; Katz, T.J.; Verbiest, T.; Elshocht, S.V.; Kuball, H.-G.; Kieswwalter, S.; Lovinger, A.J.; Persoons, A. Circular Dichroism and UV-Visible Apsorption Spectra of the Langmuir-Blodgett Films of an Aggregating Helicence. J. Am. Chem. Soc. 1998, 120, 8656-8660. [CrossRef]

43. Kuroda, R.; Harada, T.; Shindo, Y. A solid-state dedicated circular dichroism spectrophotometer: Development and application. Rev. Sci. Instr. 2001, 72, 3802-3810. [CrossRef]

44. Okamoto, Y.; Nidaira, K.; Akitsu, T. Environmental Dependence of Artifact CD Peaks of Chiral Schiff Base 3d-4f Complexes in Softmater PMMA Matrix. Int. J. Mol. Sci. 2011, 12, 6966-6979. [CrossRef] [PubMed]

45. Burck, J.; Wadhwani, P.; Fanhanel, S.; Ulrich, A.S. Oriented Circular Dichroism: A Method to Characterize Membrane-Active Peptides in Oriented Lipid Bilayers. Acc. Chem. Res. 2016, 49, 184-192. [CrossRef] [PubMed]

46. Sunaga, N.; Furuya, S.; Ito, M.; Kominato, C.; Akitsu, T. Interpretaion of chiral ordering of hybrid system of several azo dyes and chiral Schiff base Co(II) complex induced by circularly polarized light. In Computational Chemistry: Theories, Methods and Applications; Nova Science Publishers, Inc.: Houppauge, NY, USA, 2014; pp. 85-104, ISBN 978-1-63321-354-8.

47. Sunaga, N.; Kominato, C.; Ishida, N.; Ito, M.; Akitsu, T.; Konomi, T.; Katoh, M. Wavelength dependence of polarized UV light induced supramolecular orientation of azobenzene-containing organic/inorganic hybrid materials of chiral Schiff base Ni(II), $\mathrm{Cu}(\mathrm{II})$, and Zn(II) complexes. In Azobenzene: Aspects, Applications and Research; Nova Science Publishers, Inc.: Houppauge, NY, USA, 2017; pp. 117-136, ISBN 978-1-53610-673-2.

48. Sunaga, N.; Akitsu, T.; Konomi, T.; Katoh, M. The theoretical interpretation of the Linear/circularly polarized light-induced molecular orientation of the azo group-containing trans-Achiral Schiff base dinuclear complex composite material. MATEC Web Conf. 2017, 130, 07004. [CrossRef]

49. Takano, H.; Takase, M.; Sunaga, N.; Ito, M.; Akitsu, T. Viscosity and intermolecular interaction of organic/inorganic hybrid systems composed of chiral Schiff base $\mathrm{Ni}(\mathrm{II}), \mathrm{Cu}(\mathrm{II}), \mathrm{Zn}(\mathrm{II})$ complexes having long ligands, azobenzene and PMMA. Inorganics 2016, 4, 20-29. [CrossRef]

50. Akitsu, T.; Yagi, S.; Kim, S.; Yoshida, M.; Haraguchi, T. Interaction between Polarized UV Light and Hybrid Materials of Chiral Schiff base Octahedral Cu(II) complexes with Azobenzene. In The Fundamentals of Polarized Light; Terry, A., Ed.; Nova Science Publishers, Inc.: Houppauge, NY, USA, 2021; pp. 119-142, ISBN 978-1-53618-977-3.

51. Yagi, S.; Haraguchi, T.; Lo, C.-T.; Akitsu, T. Polarized Light-Induced Orientaion of Chiral Schiff Base Ni(II) and Zn(II) Complexes Having Substituted Azobenzene Moiety. In The Fundamentals of Polarized Light; Terry, A., Ed.; Nova Science Publishers, Inc.: Hauppauge, NY, USA, 2021; pp. 143-166, ISBN 978-1-53618-977-3.

52. Toyoda, K.; Miyamoto, K.; Aoki, N.; Morita, R.; Omatsu, T. Using Optical Vortex to Control the Chirality of Twisted Metal Nanostructures. Nano Lett. 2012, 12, 3645-3649. [CrossRef]

53. Yan, Z.; Scherer, N.F. Optical Vortex Induced Rotation of Silver Nanowires. J. Phys. Chem. Lett. 2013, 4, 2937-2942. [CrossRef]

54. Mechau, N.; Saphiannikova, M.; Neher, D. Dielectric and Mechanical Properties of Azobenzene Polymer Layers under Visible and Ultraviolet Irradiation. Macromolecules 2005, 38, 3894-3902. [CrossRef]

55. Barrett, C.J.; Mamiya, J.-I.; Yager, K.G.; Ikeda, T. Photo-mechanical effects in azobenzene-containing soft materials. Soft Matter 2007, 3, 1249-1261. [CrossRef] [PubMed]

56. Omatsu, T.; Miyamoto, K.; Toyoda, K.; Morita, R.; Arita, Y.; Dholakia, K. A New Twist for Materials Science: The Formation of Chiral Structures Using the Angular Momentum of Light. Adv. Opt. Mater. 2019, 7, 1801672. [CrossRef]

57. He, L.; Li, H.; Li, M. Optomechanical measurement of photon spin angular momentum and optical torque in integrated photonic devices. Sci. Adv. 2016, 6, e1600485. [CrossRef]

58. Nakatori, H.; Haraguchi, T.; Akitsu, T. Polarized light induced molecular orientation control of rigid Schiff base Ni(II), Cu(II), $\mathrm{Zn}(\mathrm{II})$ binuclear complexes as polymer composites. Symmetry 2018, 10, 147. [CrossRef]

59. Takase, M.; Yagi, S.; Haraguchi, T.; Noor, S.; Akitsu, T. Linearly Polarized UV Light-Induced Optical Anisotropy of PVA Films and Flexible Macrocycle Schiff Base Ni(II), Cu(II), Zn(II) Dinuclear Complexes. Symmetry 2018, 10, 760. [CrossRef]

60. Lavery, M.P.; Speirits, F.C.; Barnett, S.M.; Padgett, M.J. Detection of a Spinning Object Using Light's Orbital Angular Momentum. Science 2017, 341, 537-540. [CrossRef]

61. Yamazaki, S.; Haraguchi, T.; Lo, C.-T.; Akitsu, T. Polarized Light Induced Chiral Molearular Alignment of Achiral Zn(II) and Zu(II) Complexes for Depth Problem. In The Fundamentals of Polarized Light; Terry, A., Ed.; Nova Science Publishers, Inc.: Hauppauge, NY, USA, 2021; pp. 166-192, ISBN 978-1-53618-977-3.

62. Karimi, E.; Schulz, S.A.; DeLeon, I.; Qassim, H.; Upham, J.; Boyd, R.W. Generating optical orbital angular momentum at visible wavelengths using a plasmonic metasurface. Light Sci. Appl. 2014, 3, e167. [CrossRef]

63. Schmiegelow, C.T.; Schulz, J.; Kaufmann, H.; Ruster, T.; Poschinger, U.G.; Cchmidt-Kaler, F. Transfer of Optical orbital angular momentum to a bound electron. Nat. Commun. 2016, 7, 12998. [CrossRef] 
64. Bauer, T.; Banzer, P.; Karimi, E.; Oriov, S.; Rubano, A.; Marrucci, L.; Santamato, E.; Boyd, R.W.; Leuchs, G. Observation of optical polarization Mobius Strips. Science 2015, 347, 964-966. [CrossRef]

65. Eyring, H.; Liu, H.-C.; Caldwell, D. Optical Rottory Dispersion and Circular Dichroism. Chem. Rev. 1968, 68, 525-540. [CrossRef]

66. Andrews, S.S.; Tretton, J. Physical Principles of Circular Dichroism. J. Chem. Educ. 2021, 97, 4370-4376. [CrossRef]

67. Tang, Y.; Cook, T.A.; Cohen, A.E. Limits on Fluoresence Detected Circular Dichroism of Single Helicence Molecules. J. Phys. Chem. A 2009, 113, 6213-6216. [CrossRef] [PubMed]

68. Tang, Y.; Cook, T.A.; Cohen, A.E. Optically Chirality and Its Interaction with Matter. Phys. Rev. Lett. 2010, 104, 163901. [CrossRef]

69. Andrews, S.S. Using Rotational Averaging to Calculate the Bulk Response of Isotropic and Anisotropic Samples from Molecular Parameters. J. Chem. Educ. 2004, 81, 877-885. [CrossRef]

70. Sakai, K.; Nomura, K.; Yamamoto, T.; Sasaki, K. Excitation of Multipole Plasmons by Optical Vortex Beams. Sci. Rep. 2015, 5, 8431. [CrossRef] [PubMed]

71. Garoli, D.; Zilio, P.; Gorodetski, Y.; Tantussi, F.; De Angelis, F. Optical votex beam generator at nanoscale level. Sci. Rep. 2016, 6, 29547. [CrossRef] [PubMed] 